\title{
Article \\ Second-Order PDE Constrained Controlled Optimization Problems with Application in Mechanics
}

\author{
Savin Treanţă \\ Department of Applied Mathematics, University Politehnica of Bucharest, 060042 Bucharest, Romania; \\ savin.treanta@upb.ro
}

check for updates

Citation: Treanţă, S. Second-Order PDE Constrained Controlled Optimization Problems with Application in Mechanics. Mathematics 2021, 9, 1472. https:// doi.org/10.3390/math9131472

Academic Editors: Francesco Topputo and Dimplekumar N. Chalishajar

Received: 20 May 2021

Accepted: 17 June 2021

Published: 23 June 2021

Publisher's Note: MDPI stays neutral with regard to jurisdictional claims in published maps and institutional affiliations.

Copyright: (c) 2021 by the authors. Licensee MDPI, Basel, Switzerland. This article is an open access article distributed under the terms and conditions of the Creative Commons Attribution (CC BY) license (https:/ / creativecommons.org/licenses/by/ $4.0 /)$.

\begin{abstract}
The present paper deals with a class of second-order PDE constrained controlled optimization problems with application in Lagrange-Hamilton dynamics. Concretely, we formulate and prove necessary conditions of optimality for the considered class of control problems driven by multiple integral cost functionals involving second-order partial derivatives. Moreover, an illustrative example is provided to highlight the effectiveness of the results derived in the paper. In the final part of the paper, we present an algorithm to summarize the steps for solving a control problem such as the one investigated here.
\end{abstract}

Keywords: multi-time controlled second-order Lagrangian; Euler-Lagrange equations; second-order PDE constraints; multiple integral functional

\section{Introduction}

Calculus of Variations and Optimal Control Theory are two mathematical fields with strong and important connections, with significant applications in applied sciences, engineering, data analysis, and classification. Over time, many researchers have investigated these areas and the connections between them, obtaining remarkable results. In this regard, we mention only the works of Friedman [1], Hestenes [2], Kendall [3], Udrişte [4], Petrat and Tumulka [5], Treanţă [6], Deckert and Nickel [7], and Olteanu and Treanţă [8]. In fact, before, we wanted to specify in particular the research papers that dealt with problems in several time variables. In the last decade, the study of multi-dimensional optimization problems (with important applications in various branches of mathematical sciences, engineering design, portfolio selection, game theory, decision problems in management science, data analysis, web access problems, query optimization in databases, and so forth), have been studied by Mititelu and Treanţă [9], Treanţă [10-17], Jayswal et al. [18], and Treanţă [19-21]. More precisely, some classes of variational problems driven by multiple and path-independent curvilinear integral cost functionals with isoperimetric and mixed constraints involving PDEs of $m$-flow type and partial differential inequations have been introduced and studied. In addition, the isoperimetric constrained optimization problems have been of particular interest to many researchers due to their importance in the applied sciences. We mention, for example, the research works of Schmitendorf [22,23], Forster and Long [24], and Treanţă [13]. More specifically, by using the Pontryagin's principle, Schmitendorf [22] studied the necessary conditions of optimality associated with a class of control problems involving isoperimetric and inequality constraints at the terminal time. Later, Forster and Long [24] (see also Schmitendorf [23]), by considering an alternative transformation technique, established the associated necessary optimality conditions for the same optimization problem. Quite recently, Treanţă [13] has focused on the optimization of some simple, multiple or curvilinear integral functionals (governed by second-order Lagrangians) subject to ODE, PDE or isoperimetric constraints. In addition, Pascalis et al. [25] used genetic algorithms in order to theoretically design a range of phononic media that can act to prevent or ensure antiplane elastic wave propagation over a specific range of low frequencies, with each case corresponding to a specific pre-stress level. 
In the present paper, inspired by the previous research in this field, we investigate a new class of PDE constrained optimization problems driven by multiple integral cost functionals which involve second-order partial derivatives. More precisely, the proper motivation for studying such issues is:

(i) Considering the bi-temporal optimal problem with pointwise state constraints:

$$
\min _{x, v} \frac{1}{2} \int_{\Omega_{t_{0}, t_{1}}}\left(x(t)-\sin \left(2 \pi t^{1} t^{2}\right)\right)^{2} d t^{1} d t^{2}+\frac{\alpha}{2} \int_{\Omega_{t_{0}, t_{1}}} v^{2}(t) d t^{1} d t^{2}
$$

subject to

$$
-\Delta x(t)=v(t), t \in \Omega_{t_{0}, t_{1}} ; \quad x(t)=0, t \in \partial \Omega_{t_{0}, t_{1}} .
$$

This optimization problem has also been studied in Udrişte and Matei [26] by applying a simplified multi-time maximum principle.

(ii) (Neumann boundary control): Find a control function $v \in L^{2}(\Gamma)$ that minimizes the cost functional

$$
J(v(\cdot))=\frac{1}{2} \int_{\Omega}\left(z(t)-z_{d}\right)^{2} d t^{1} \cdots d t^{m}+\frac{\beta}{2} \int_{\Gamma} v^{2}(t) d s
$$

where $(z, v)$ satisfies $-\Delta z+z=f$ in $\Omega$ and $\frac{\partial z}{\partial n}=v$ on $\Gamma$, the function $f$ is a given source term, the function $v$ is a control variable, and $\Omega$ is a bounded domain in $R^{m}$ with a boundary $\Gamma$ of class $C^{2}$. Since the term $\frac{\beta}{2} \int_{\Gamma} v^{2}(t) d s$ (see $\beta>0$ ) is proportional to the consumed energy, the minimizing of $J$ is a compromise between the energy consumption and finding $v$ so that the distribution $z$ is close to the desired profile $z_{d}$. We say that the control function is a boundary control because the control acts on $\Gamma$.

The foregoing two examples of optimal control problems (involving second-order partial derivatives) have been taken from the literature. For more details, other examples, and different points of view, we refer to Raymond [27] and Udrişte and Matei [26].

The mathematical framework developed in this paper is more general than in Hestenes [2], Schmitendorf [22], Udrişte and Ţevy [4], or Treanţă [13], both by the presence of controlled multiple integrals and by the inclusion of the new proof associated with the main result and the second-order partial derivatives. Therefore, this paper can be seen as a fundamental work for researchers in the field of applied sciences, mechanics, data analysis, and classification, where second-order PDEs (partial speed-acceleration constraints) are involved. It should also be mentioned that a simplified language is used, specific to applied mathematics, and readers of pure mathematics are referred to the bibliography. The main purpose is to expose ideas stripped of excessive formalizations, which have a great impact on the reader's understanding of the natural phenomena encountered in engineering and economics. In this sense, mathematical modeling must be only the scientific support for the intelligent presentation of the real world and not the abstract notions specific to pure mathematics.

The paper is organized as follows. In Section 2, we introduce the optimization problem under study and formulate the main result of this paper, that is, Theorem 1. This theorem provides the necessary optimality conditions for the considered second-order PDE-constrained control problem. Moreover, the final part of this section includes an illustrative application and an algorithm. Finally, Section 3 presents the conclusions of the paper. 


\section{Second-Order PDE Constrained Controlled Optimization Problem}

We consider a $C^{3}$-class function $\mathcal{L}\left(s(t), s_{\gamma}(t), s_{\alpha \beta}(t), v(t), t\right)$, called multi-time controlled second-order Lagrangian, where $t=\left(t^{\alpha}\right)=\left(t^{1}, \cdots, t^{m}\right) \in \Lambda_{t_{0}, t_{1}} \subset \mathbb{R}_{+}^{m}, s=\left(s^{i}\right)=$ $\left(s^{1}, \cdots, s^{n}\right): \Lambda_{t_{0}, t_{1}} \rightarrow \mathbb{R}^{n}$ is the state function of class $C^{4}$, and $v=\left(v^{\vartheta}\right)=\left(v^{1}, \cdots, v^{k}\right):$ $\Lambda_{t_{0}, t_{1}} \rightarrow \mathbb{R}^{k}$ is the control function (a piecewise continuous function). We also consider $s_{\alpha}(t):=\frac{\partial s}{\partial t^{\alpha}}(t), s_{\alpha \beta}(t):=\frac{\partial^{2} s}{\partial t^{\alpha} \partial t^{\beta}}(t), \alpha, \beta \in\{1, \ldots, m\}$, and $\Lambda_{t_{0}, t_{1}}=\left[t_{0}, t_{1}\right]$ (multi-time interval in $\mathbb{R}_{+}^{m}$ ) is a hyper-parallelepiped generated by $t_{0}, t_{1} \in \mathbb{R}_{+}^{m}$ (diagonally opposite points).

Second-order PDE constrained controlled optimization problem. Find $\left(s^{*}, v^{*}\right)$ that minimizes the following multiple integral cost functional

$$
L(s(\cdot), v(\cdot))=\int_{\Lambda_{t_{0}, t_{1}}} \mathcal{L}\left(s(t), s_{\gamma}(t), s_{\alpha \beta}(t), v(t), t\right) d t^{1} \cdots d t^{m}
$$

among all the functions $(s, v)$ that satisfy

$$
s\left(t_{0}\right)=s_{0}, \quad s\left(t_{1}\right)=s_{1}, \quad s_{\gamma}\left(t_{0}\right)=\tilde{s}_{\gamma 0}, \quad s_{\gamma}\left(t_{1}\right)=\tilde{s}_{\gamma 1},
$$

or

$$
\left.s(t)\right|_{\partial \Lambda_{t_{0}, t_{1}}}=\text { given, }\left.s_{\gamma}(t)\right|_{\partial \Lambda_{t_{0}, t_{1}}}=\text { given }
$$

and the controlled second-order PDE constraints (partial speed-acceleration constraints) defined as follows:

$$
g_{\zeta}^{a}\left(s(t), s_{\gamma}(t), s_{\alpha \beta}(t), v(t), t\right)=0, \quad a=1,2, \cdots, r \leq n, \zeta=1,2, \cdots, l \leq m .
$$

In order to investigate the above controlled optimization problem (1), associated with the aforementioned controlled second-order PDE constraints, we introduce the Lagrange multiplier $b=\left(p_{a}^{\zeta}(t)\right)$ and build a new multi-time controlled second-order Lagrangian (see summation over the repeated indices, Einstein summation)

$$
\begin{gathered}
\mathcal{L}_{1}\left(s(t), s_{\gamma}(t), s_{\alpha \beta}(t), v(t), b(t), t\right)=\mathcal{L}\left(s(t), s_{\gamma}(t), s_{\alpha \beta}(t), v(t), t\right) \\
+p_{a}^{\zeta}(t) g_{\zeta}^{a}\left(s(t), s_{\gamma}(t), s_{\alpha \beta}(t), v(t), t\right),
\end{gathered}
$$

that changes the initial controlled optimization problem (with second-order PDE constraints) into a partial speed-acceleration unconstrained controlled optimization problem

$$
\begin{gathered}
\min _{(s(\cdot), v(\cdot), b(\cdot))} \int_{\Lambda_{t_{0}, t_{1}}} \mathcal{L}_{1}\left(s(t), s_{\gamma}(t), s_{\alpha \beta}(t), v(t), b(t), t\right) d t^{1} \cdots d t^{m} \\
s\left(t_{q}\right)=s_{q}, \quad s_{\gamma}\left(t_{q}\right)=\tilde{s}_{\gamma q}, \quad q=0,1 .
\end{gathered}
$$

In accordance with Lagrange theory, under suitable constraint qualifications, an extreme point of (1) is found among the extreme points of (2).

To formulate the necessary optimality conditions associated with the aforementioned controlled optimization problem, we shall introduce the Saunders's multi-index notation (see Saunders [28], Treanţă [29]).

The main result of this paper is provided by the following theorem. It formulates the necessary optimality conditions for the second-order PDE constrained optimization problem (1).

Theorem 1. If $\left(s^{*}(\cdot), v^{*}(\cdot), b^{*}(\cdot)\right)$ is a solution for (2), then

$$
\left(s^{*}(\cdot), v^{*}(\cdot), b^{*}(\cdot)\right)
$$


is a solution of the following system of Euler-Lagrange PDEs

$$
\begin{gathered}
\frac{\partial \mathcal{L}_{1}}{\partial s^{i}}-D_{\gamma} \frac{\partial \mathcal{L}_{1}}{\partial s_{\gamma}^{i}}+\frac{1}{\mu(\alpha, \beta)} D_{\alpha \beta}^{2} \frac{\partial \mathcal{L}_{1}}{\partial s_{\alpha \beta}^{i}}=0, \quad i=\overline{1, n} \\
\frac{\partial \mathcal{L}_{1}}{\partial v^{\vartheta}}-D_{\gamma} \frac{\partial \mathcal{L}_{1}}{\partial u_{\gamma}^{\vartheta}}+\frac{1}{\mu(\alpha, \beta)} D_{\alpha \beta}^{2} \frac{\partial \mathcal{L}_{1}}{\partial u_{\alpha \beta}^{\vartheta}}=0, \quad \vartheta=\overline{1, k} \\
\frac{\partial \mathcal{L}_{1}}{\partial p_{a}^{\zeta}}-D_{\gamma} \frac{\partial \mathcal{L}_{1}}{\partial p_{a, \gamma}^{\zeta}}+\frac{1}{\mu(\alpha, \beta)} D_{\alpha \beta}^{2} \frac{\partial \mathcal{L}_{1}}{\partial p_{a, \alpha \beta}^{\zeta}}=0, \quad a=\overline{1, r}, \zeta=\overline{1, l},
\end{gathered}
$$

where $p_{a, \gamma}^{\zeta}:=\frac{\partial p_{a}^{\zeta}}{\partial t^{\gamma}}, p_{a, \alpha \beta}^{\zeta}:=\frac{\partial^{2} p_{a}^{\zeta}}{\partial t^{\alpha} \partial t^{\beta}}, u_{\alpha \beta}^{\vartheta}:=\frac{\partial^{2} v^{\vartheta}}{\partial t^{\alpha} \partial t^{\beta}}, \alpha, \beta, \gamma \in\{1,2, \ldots, m\}$.

Proof. Consider that $(s(t), v(t), b(t))$ is a solution for (2). By considering the variations $s(t)+\varepsilon \mathrm{h}(t)$, with $\left.\mathrm{h}(t)\right|_{\partial \Lambda_{t_{0}, t_{1}}}=0,\left.\mathrm{~h}_{\eta}(t)\right|_{\partial \Lambda_{t_{0}, t_{1}}}=0, \eta \in\{1,2, \ldots, m\}$ (see $\mathrm{h}_{\eta}:=\frac{\partial \mathrm{h}}{\partial t^{\eta}}$ ), $b(t)+\varepsilon f(t)$, with $\left.f(t)\right|_{\partial \Lambda_{t_{0}, t_{1}}}=0$, and $v(t)+\varepsilon \mathrm{m}(t)$, with $\left.\mathrm{m}(t)\right|_{\partial \Lambda_{t_{0}, t_{1}}}=0$, the controlled multiple integral cost functional becomes a function depending on $\varepsilon$. Consequently, it is a controlled multiple integral with parameter

$$
\begin{gathered}
I(\varepsilon)=\int_{\Lambda_{t_{0}, t_{1}}} \mathcal{L}_{1}\left(s(t)+\varepsilon \mathrm{h}(t), s_{\gamma}(t)+\varepsilon \mathrm{h}_{\gamma}(t), s_{\alpha \beta}(t)+\varepsilon \mathrm{h}_{\alpha \beta}(t), v(t)+\varepsilon \mathrm{m}(t),\right. \\
b(t)+\varepsilon f(t), t) d t^{1} \cdots d t^{m} .
\end{gathered}
$$

By using the hypothesis, we obtain

$$
\begin{gathered}
\left.\frac{d}{d \varepsilon} I(\varepsilon)\right|_{\varepsilon=0}=\int_{\Lambda_{t_{0}, t_{1}}}\left(\frac{\partial \mathcal{L}_{1}}{\partial s^{j}} \mathrm{~h}^{j}+\frac{\partial \mathcal{L}_{1}}{\partial s_{\gamma}^{j}} \mathrm{~h}_{\gamma}^{j}+\frac{1}{\mu(\alpha, \beta)} \frac{\partial \mathcal{L}_{1}}{\partial s_{\alpha \beta}^{j}} \mathrm{~h}_{\alpha \beta}^{j}+\frac{\partial \mathcal{L}_{1}}{\partial v^{\vartheta}} \mathrm{m}^{\vartheta}\right. \\
\left.+\frac{\partial \mathcal{L}_{1}}{\partial p_{a}^{\zeta}} \mathrm{f}_{\zeta}^{a}\right) d t^{1} \cdots d t^{m} \\
=B T+\int_{\Lambda_{t_{0}, t_{1}}}\left(\frac{\partial \mathcal{L}_{1}}{\partial s^{j}}-D_{\gamma} \frac{\partial \mathcal{L}_{1}}{\partial s_{\gamma}^{j}}+\frac{1}{\mu(\alpha, \beta)} D_{\alpha \beta}^{2} \frac{\partial \mathcal{L}_{1}}{\partial s_{\alpha \beta}^{j}}\right) \mathrm{h}^{j} d t^{1} \cdots d t^{m} \\
+\int_{\Lambda_{t_{0}, t_{1}}}\left(\frac{\partial \mathcal{L}_{1}}{\partial v^{\vartheta}}-D_{\gamma} \frac{\partial \mathcal{L}_{1}}{\partial u_{\gamma}^{\vartheta}}+\frac{1}{\mu(\alpha, \beta)} D_{\alpha \beta}^{2} \frac{\partial \mathcal{L}_{1}}{\partial u_{\alpha \beta}^{\vartheta}}\right) \mathrm{m}^{\vartheta} d t^{1} \cdots d t^{m} \\
+\int_{\Lambda_{t_{0}, t_{1}}}\left(\frac{\partial \mathcal{L}_{1}}{\partial p_{a}^{\zeta}}-D_{\gamma} \frac{\partial \mathcal{L}_{1}}{\partial p_{a, \gamma}^{\zeta}}+\frac{1}{\mu(\alpha, \beta)} D_{\alpha \beta}^{2} \frac{\partial \mathcal{L}_{1}}{\partial p_{a, \alpha \beta}^{\zeta}}\right) \mathrm{f}_{\zeta}^{a} d t^{1} \cdots d t^{m}=0 .
\end{gathered}
$$

In addition, by using the formula of integration by parts, it results that

$$
\begin{gathered}
\frac{\partial \mathcal{L}_{1}}{\partial s_{\gamma}^{j}} \mathrm{~h}_{\gamma}^{j}=-\mathrm{h}^{j} D_{\gamma} \frac{\partial \mathcal{L}_{1}}{\partial s_{\gamma}^{j}}+D_{\gamma}\left(\frac{\partial \mathcal{L}_{1}}{\partial s_{\gamma}^{j}} \mathrm{~h}^{j}\right), \\
\frac{1}{\mu(\alpha, \beta)} \frac{\partial \mathcal{L}_{1}}{\partial s_{\alpha \beta}^{j}} \mathrm{~h}_{\alpha \beta}^{j}=\frac{1}{\mu(\alpha, \beta)}\left[\mathrm{h}^{j} D_{\alpha \beta}^{2} \frac{\partial \mathcal{L}_{1}}{\partial s_{\alpha \beta}^{j}}-D_{\alpha}\left(\mathrm{h}^{j} D_{\beta} \frac{\partial \mathcal{L}_{1}}{\partial s_{\alpha \beta}^{j}}\right)+D_{\beta}\left(\frac{\partial \mathcal{L}_{1}}{\partial s_{\alpha \beta}^{j}} \mathrm{~h}_{\alpha}^{j}\right)\right]
\end{gathered}
$$

and taking into account the divergence formula, the boundary terms BT (given below) vanish (see $\left.\mathrm{h}\right|_{\partial \Lambda_{t_{0}, t_{1}}}=\left.\mathrm{m}\right|_{\partial \Lambda_{t_{0}, t_{1}}}=\left.\mathrm{f}\right|_{\partial \Lambda_{t_{0}, t_{1}}}=0,\left.\mathrm{~h}_{\eta}\right|_{\partial \Lambda_{t_{0}, t_{1}}}=0, \mathbf{n}^{\xi}(t)=$ the unit normal vector on $\partial \Lambda_{t_{0}, t_{1}}$, and $\delta_{\nu \xi}=$ the Kronecker's synmbol), 


$$
\begin{gathered}
\int_{\Lambda_{t_{0}, t_{1}}} D_{\gamma}\left(\frac{\partial \mathcal{L}_{1}}{\partial s_{\gamma}^{j}} h^{j}\right) d t^{1} \cdots d t^{m}=\int_{\partial \Lambda_{t_{0}, t_{1}}} \delta_{\nu \xi} \frac{\partial \mathcal{L}_{1}}{\partial s_{v}^{j}} h^{j} \mathbf{n}^{\xi} d \sigma, \\
\int_{\Lambda_{t_{0}, t_{1}}} D_{\alpha}\left(h^{j} D_{\beta} \frac{\partial \mathcal{L}_{1}}{\partial s_{\alpha \beta}^{j}}\right) d t^{1} \ldots d t^{m}=\int_{\partial \Lambda_{t_{0}, t_{1}}} \delta_{\nu \xi} D_{\beta} \frac{\partial \mathcal{L}_{1}}{\partial s_{\nu \beta}^{j}} h^{j} \mathbf{n}^{\xi} d \sigma, \\
\int_{\Lambda_{t_{0}, t_{1}}} D_{\beta}\left(\frac{\partial \mathcal{L}_{1}}{\partial s_{\alpha \beta}^{j}} h_{\alpha}^{j}\right) d t^{1} \ldots d t^{m}=\int_{\partial \Lambda_{t_{0}, t_{1}}} \delta_{\nu \xi} \frac{\partial \mathcal{L}_{1}}{\partial s_{\alpha \nu}^{j}} \mathrm{~h}_{\alpha}^{j} \mathbf{n}^{\xi} d \sigma .
\end{gathered}
$$

Now, since the "small" variations $h, f, m$ were taken arbitrarily, by using a fundamental lemma of variational calculus, the relation (3) leads to the system of partial differential equations formulated in theorem, and the proof is complete.

Remark 1. The system of Euler-Lagrange PDEs given in Theorem 1 can be reformulated as follows

$$
\begin{gathered}
\frac{\partial \mathcal{L}_{1}}{\partial s^{i}}-D_{\gamma} \frac{\partial \mathcal{L}_{1}}{\partial s_{\gamma}^{i}}+\frac{1}{\mu(\alpha, \beta)} D_{\alpha \beta}^{2} \frac{\partial \mathcal{L}_{1}}{\partial s_{\alpha \beta}^{i}}=0, \quad i=\overline{1, n} \\
\frac{\partial \mathcal{L}_{1}}{\partial v^{\vartheta}}-D_{\gamma} \frac{\partial \mathcal{L}_{1}}{\partial u_{\gamma}^{\vartheta}}+\frac{1}{\mu(\alpha, \beta)} D_{\alpha \beta}^{2} \frac{\partial \mathcal{L}_{1}}{\partial u_{\alpha \beta}^{\vartheta}}=0, \quad \vartheta=\overline{1, k} \\
g_{\zeta}^{a}\left(s(t), s_{\gamma}(t), s_{\alpha, \beta}(t), v(t), t\right)=0, \quad a=1,2, \cdots, r \leq n, \zeta=1,2, \cdots, l \leq m .
\end{gathered}
$$

Example 1. Let the double integral cost functional be given by

$$
L(s(\cdot), v(\cdot))=\int_{[0,1]^{2}}\left(s^{2}(t)+v^{2}(t)\right) d t^{1} d t^{2} .
$$

Find the extremals of $L(s(\cdot), v(\cdot))$ subject to $s_{t^{1}}(t)+s_{t^{2}}(t)=0$ and the boundary conditions $s(0,0)=s(1,1)=0$.

Solution. The associated auxiliary controlled Lagrangian is given by

$$
\mathcal{L}_{1}=s^{2}(t)+v^{2}(t)+b(t)\left(s_{t^{1}}(t)+s_{t^{2}}(t)\right)
$$

and the extremals are characterized by the following PDEs

$$
\begin{gathered}
2 s-\frac{\partial b}{\partial t^{1}}-\frac{\partial b}{\partial t^{2}}=0, \\
2 u=0, \\
s_{t^{1}}(t)+s_{t^{2}}(t)=0,
\end{gathered}
$$

implying that $\left(s^{*}, v^{*}\right)=\left(s^{*}, 0\right)$ is the optimal solution of the considered PDE constrained optimization problem, corresponding to the Lagrange multiplier $b$ satisfying $p_{\left(t^{1}\right)^{2}}(t)+2 p_{t^{1} t^{2}}(t)+$ $p_{\left(t^{2}\right)^{2}}(t)=0$.

The intention of the following algorithm (see Algorithm 1) is to summarize the steps for solving a control problem such as the one investigated here. More precisely, for a multiple integral cost functional and a set of mixed (boundary conditions and second-order PDE) restrictions, the goal is to find $\left(s^{\star}, v^{\star}\right)$ (which fulfils the set of mixed constraints) such that $L\left(s^{\star}, v^{\star}\right) \leq L(s, v)$ for all feasible points $(s, v)$. In this direction, we begin with a feasible point $(s, v)$. If $(s, v)$ satisfies the necessary conditions of optimality formulated in Theorem 1, then the "Generating Stage" (see below) is completed and we go to the next step; else, the algorithm stops. For $\left(s^{\star}, v^{\star}\right)$ obtained in "Generating Stage", if $L\left(s^{\star}, v^{\star}\right) \leq L(s, v)$ 
is true, for all feasible points $(s, v)$, then $\left(s^{\star}, v^{\star}\right)$ is an optimal solution for the considered problem; else, the algorithm stops.

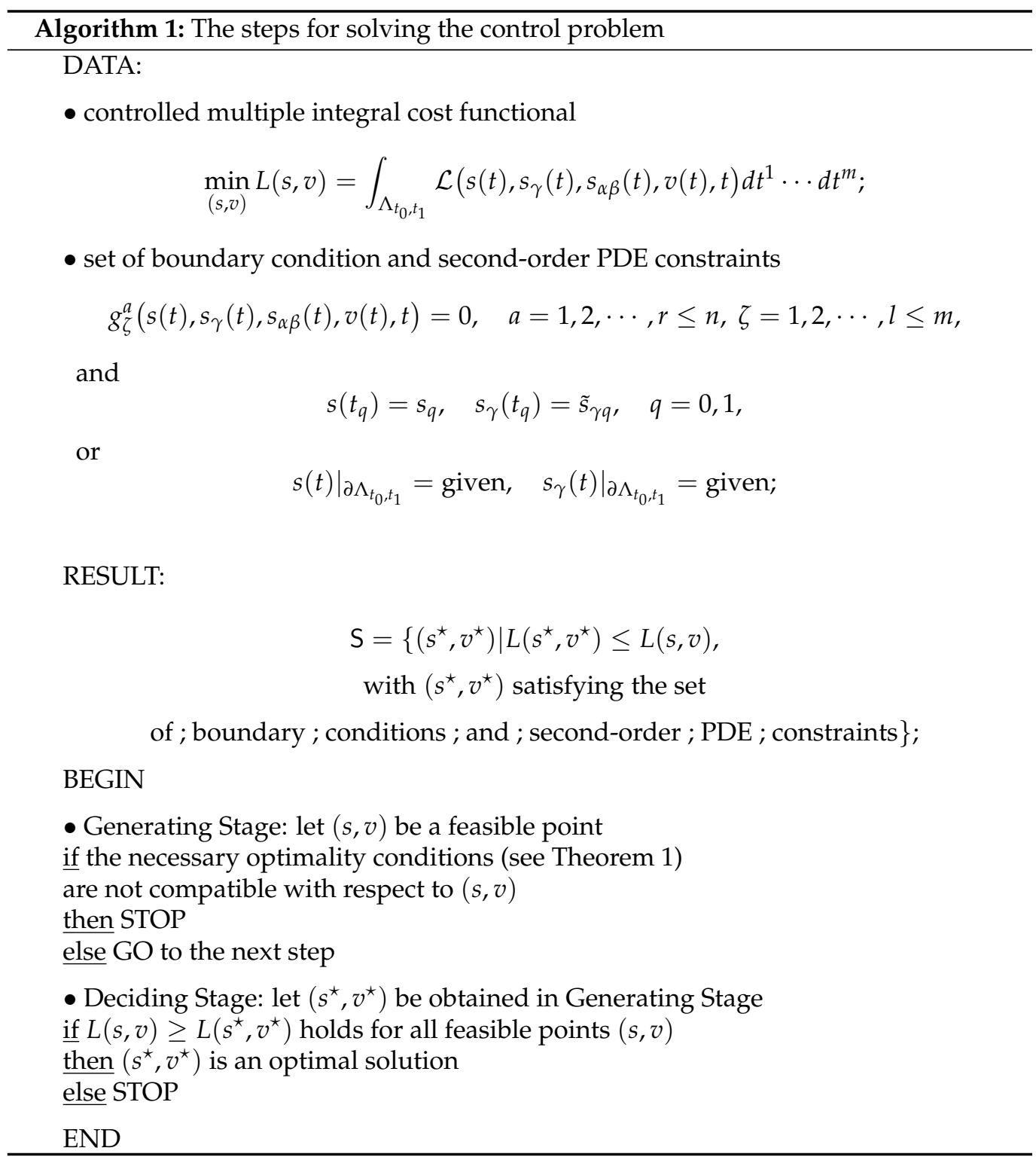

\section{Conclusions}

In this paper, we have introduced and investigated a class of second-order PDE constrained controlled optimization problems with application in Lagrange-Hamilton dynamics. More specifically, necessary conditions of optimality were established for the considered class of variational problems driven by multiple integral cost functionals involving second-order partial derivatives. Moreover, the theoretical results are accompanied by an illustrative example. Finally, we have presented an algorithm to synthesize the steps for solving a controlled optimization problem such as the one investigated in the paper.

A new direction of research, on the considered class of control problems introduced in this paper, is given, for example, by the study of well-posedness and well-posedness in the generalized sense.

Funding: This research received no external funding.

Institutional Review Board Statement: Not applicable.

Informed Consent Statement: Not applicable. 


\section{Data Availability Statement: Not applicable.}

Acknowledgments: The author would like to thank anonymous referees for their careful reading and constructive suggestions that substantially improve the revision of the manuscript.

Conflicts of Interest: The author declares no conflict of interest.

\section{References}

1. Friedman, A. The Cauchy problem in several time variables. J. Math. Mech. (Indiana Univ. Math. J.) 1962, 11, 859-889.

2. Hestenes, M. Calculus of Variations and Optimal Control Theory; John Wiley and Sons: New York, NY, USA,1966.

3. Kendall, W.S. Contours of Brownian processes with several-dimensional times. Probab. Theory Relat. Fields 1980, 52, $267-276$. [CrossRef]

4. Udrişte, C.; Ţevy, I. Multi-time Euler-Lagrange-Hamilton theory. WSEAS Trans. Math. 2007, 6, 701-709.

5. Petrat, S.; Tumulka, R. Multi-time wave functions for quantum field theory. Ann. Phys. 2014, 345, 17-54. [CrossRef]

6. Treanţă, S. PDEs of Hamilton-Pfaff type via multi-time optimization problems. UPB Sci. Bull. Ser. A 2014, 76, 163-168.

7. Deckert, D.A.; Nickel, L. Consistency of multi-time Dirac equations with general interaction potentials. J. Math. Phys. 2016, 57, 072301. [CrossRef]

8. Olteanu, O.; Treanţă, S. Convexity, Optimization and Approximation, with some Applications; LAP Lambert Academic Publishing: Saarbrucken, Germany, 2018; ISBN 978-613-9-87683-9.

9. Mititelu, Ş.; Treanţă, S.Efficiency conditions in vector control problems governed by multiple integrals. J. Appl. Math. Comput. 2018, 57, 647-665. [CrossRef]

10. Treanţă, S. On a new class of vector variational control problems. Numer. Funct. Anal. Optim. 2018, 39, 1594-1603. [CrossRef]

11. Treanţă, S. KT-geodesic pseudoinvex control problems governed by multiple integrals. J. Nonlinear Convex Anal. $2019,20,73-84$.

12. Treanţă, S. Saddle-point optimality criteria in modified variational control problems with PDE constraints. Optim. Control. Appl. Methods 2020, 41, 1160-1175. [CrossRef]

13. Treanţă, S. Constrained variational problems governed by second-order Lagrangians. Appl. Anal. 2020, 99, 1467-1484. [CrossRef]

14. Treanţă, S.; Arana-Jiménez, M.; Antczak, T. A necessary and sufficient condition on the equivalence between local and global optimal solutions in variational control problems. Nonlinear Anal. Theory Methods Appl. 2020, 191, UNSP 111640. [CrossRef]

15. Treanţă, S. Efficiency in generalized V-KT-pseudoinvex control problems. Int. J. Control. 2020, 93, 611-618. [CrossRef]

16. Treanţă, S. Characterization of efficient solutions for a class of PDE-constrained vector control problems. Numer. Algebr. Control. Optim. 2020, 10, 93-106. [CrossRef]

17. Treanţă, S. On modified interval-valued variational control problems with first-order PDE constraints. Symmetry 2020, $12,472$. [CrossRef]

18. Jayswal, A. An exact 11 penalty function method for multi-dimensional first-order PDE constrained control optimization problem. Eur. J. Control. 2020, 52, 34-41. [CrossRef]

19. Treanţă, S. Duality theorems for $(\rho, \psi, d)$-quasiinvex multiobjective optimization problems with interval-valued components. Mathematics 2021, 9, 894. [CrossRef]

20. Treanţă, S. Saddle-point optimality criteria involving $(\rho, b, d)$-invexity and $(\rho, b, d)$-pseudoinvexity in interval-valued optimization problems. Int. J. Control. 2020. [CrossRef]

21. Treanţă, S. Efficiency in uncertain variational control problems. Neural Comput. Appl. 2021, 33, 5719-5732. [CrossRef]

22. Schmitendorf, W.E. Pontryagin's principle for problems with isoperimetric constraints and for problems with inequality terminal constraints. J. Optim. Theory Appl. 1976, 18, 561-567. [CrossRef]

23. Schmitendorf, W.E. Pontryagin's principle for problems with isoperimetric constraints and for problems with inequality terminal constraints: Reply. J. Optim. Theory Appl. 1978, 25, 323. [CrossRef]

24. Forster, B.A.; Long, N.V. Pontryagin's principle for problems with isoperimetric constraints and for problems with inequality terminal constraints: Comment. J. Optim. Theory Appl. 1978, 25, 317-322. [CrossRef]

25. Pascalis, R.D.; Donateo, T.; Ficarella, A.; Parnell, W.J. Optimal design of phononic media through genetic algorithm-informed pre-stress for the control of antiplane wave propagation. Extrem. Mech. Lett. 2020, 40, 100896. [CrossRef]

26. Udrişte, C.; Matei, L. Lagrange-Hamilton Theories; Monographs and Textbooks 8; Geometry Balkan Press: Bucharest, Romania, 2008. (In Romanian)

27. Raymond, J.P. Optimal Control of Partial Differential Equations; Université Paul Sabatier: Toulouse, France, 2010.

28. Saunders, D.J. The Geometry of Jet Bundles; London Math. Soc. Lecture Notes Series 142; Cambridge University Press: Cambridge, UK, 1989.

29. Treanţă, S. On a Class of Isoperimetric Constrained Controlled Optimization Problems. Axioms 2021, 10, 112. [CrossRef] 